\title{
纳米 $\mathrm{ZnO}$ 的表面增强拉曼散射效应来源研究
}

\author{
倪宇欣张晨杰袁亚仙* 徐敏敏 姚建林* \\ (苏州大学材料与化学化工学部 苏州 215123)
}

\begin{abstract}
摘要 表面增强拉曼光谱(SERS)的广泛应用源于优良的基底, 目前主要局限在粗粘贵金属及胶体纳米颗粒材料. 而半 导体的光谱高稳定性和再现性, 使其成为制备 SERS 基底的新型材料, 但对于其 SERS 增强机理的研究仍存在极大挑 战. 本工作以典型的形貌新颖、尺寸均一的扫帚状 $\mathrm{n}$ 型纳米半导体 $\mathrm{ZnO}$ 为 SERS 基底材料, 通过调节激发光的波长和 选用具有不同对位取代基的对硝基苯硫酚(PNTP)、苯硫酚(TP)、对氨基苯硫酚(PATP)为探针分子, 系统地研究了纳米 $\mathrm{ZnO}$ 的 SERS 增强行为, 估算了其表面增强因子 $(E F)$, 分离了化学增强作用中非共振增强效应和电荷转移效应对 SERS 的贡献. 研究表明三种分子在不同激发光作用下的增强因子为 10 至 35, 其中 PNTP 分子约 10 倍的增强主要来自于因 吸附而造成极化率变化的非共振增强效应, TP 和 PATP 分子 20 35 倍的增强则是由非共振增强效应与光子驱动电荷转 移效应共同作用所致, 光子能量越高, SERS 增强效应越强. 且因分子与 $\mathrm{ZnO}$ 间电荷转移的速率较慢导致 $\mathrm{ZnO}$ 表面电荷 转移增强效应较贵金属低 1 2 个数量级. 本研究结果为新型半导体 SERS 基底的制备及调控提供了新思路.

关键词 表面增强拉曼光谱; $\mathrm{ZnO}$; 表面增强因子; 非共振增强; 电荷转移增强
\end{abstract}

\section{Determination on Origination of Surface Enhanced Raman Scattering Effect on Nano ZnO Substrate}

\author{
Ni, Yuxin Zhang, Chenjie Yuan, Yaxian* Xu, Minmin Yao, Jianlin* \\ (College of Chemistry, Chemical Engineering and Materials Science, Soochow University, Suzhou 215123)
}

\begin{abstract}
The promising application of surface-enhanced Raman spectroscopy (SERS) was definitely based on the high quality substrates which were restricted to the rough noble metals and colloidal nanoparticle materials. However, semiconductor has become a potential substrate for the SERS investigation due to its high stability and reproducibility. It remains significant challenges in interpreting the enhancement mechanisms. Herein, broom-like $\mathrm{ZnO}$ nanoparticles with novel morphology and uniform size was prepared by pyrolysis of $\left(\mathrm{CH}_{3} \mathrm{COO}\right)_{2} \mathrm{Zn}$. By using $p$-nitrophenylthiophenol (PNTP), phenylthiophenol (TP) and $p$-aminophenylthiophenol (PATP) as probe molecules, the SERS effect on ZnO surfaces was systematically studied under the irradiation of excitation lines with the wavelength of $532 \mathrm{~nm}$ and $638 \mathrm{~nm}$. The different substituents in $p$-position of TP allowed to change the energy levels by the electron withdrawing or donating group, it was beneficial to match the energy level gap between the probe molecules and semiconductor for triggering the photon driven charge transfer. The surface enhancement factor $(E F)$ of broom-like $\mathrm{ZnO}$ nanoparticles were estimated accordingly, and the contribution of non-resonance and charge transfer to SERS effect was distinguished. The results demonstrated that the surface enhancement factor was about 10 to 35 times depending on the probe molecules and excitation wavelengths. Therefore, the different enhancement origination contributed to the different molecules on the $\mathrm{ZnO}$ substrate. For the TP and PATP, the charge transfer from the HOMO level of molecule to $\mathrm{CB}$ of $\mathrm{ZnO}$ was achieved by the assistance of the laser photon with the appropriate energy. Moreover, the higher energy of the photon is, the stronger the SERS enhancement effect. As for the PNTP, the photon driven charge transfer was absent due to the significant change of the HOMO and LUMO level caused by the electron withdrawing group of $\mathrm{NO}_{2}$. It revealed that the enhancement effect of PNTP molecule about 10 times was contributed by the non-resonance enhancement mechanism which was mainly due to the changes in the polarizability caused by the chemical adsorption. Comparing to the noble metal surface, the enhancement of charge transfer on $\mathrm{ZnO}$ was decreased with $1 \sim 2$ orders of magnitude. The relatively lower rate of charge transfer in semiconductor resulted in the decrease of the charge transfer enhancement. The preliminary studies provided a novel approach for the preparation and regulation of new semiconductor SERS substrates.
\end{abstract}

Keywords surface-enhanced Raman Spectroscopy (SERS); ZnO; surface enhancement factor $(E F)$; non-resonance enhancement; photon-driven charge transfer enhancement

\footnotetext{
*E-mail: yuanyaxian@suda.edu.cn; jlyao@suda.edu.cn; Tel.: 0512-65880359; Fax: 0512-65880089

Received April 30, 2019; published June 20, 2019.

Supporting information for this article is available free of charge via the Internet at http://sioc-journal.cn.

Project supported by the National Natural Science Foundation of China (Nos. 21773166, 21673152).

项目受国家自然科学基金(Nos. 21773166, 21673152)资助.
} 


\section{1 引言}

表面增强拉曼光谱(surface-enhanced Raman spectroscopy, SERS)因灵敏度高、检测速度快、能在分子水 平提供表面吸附物种丰富的特征指纹信息而成为广受 表面科学家青崃的表征技术之一 ${ }^{[1,2]}$. 事实上, 巨大的表 面增强效应主要来自于金属中自由电子的集体振荡与 外加光电场作用产生的表面等离激元共振 (surface plasmon resonance, SPR ${ }^{[3,4]}$, 其可以引起金属表面局域 电磁场的增强以及耦合作用, 增强物质光激发、光吸收、 光散射等过程 ${ }^{[5]}$, 该效应称为电磁场增强 (electromagnetic enhancement, EM), 其可产生 $10^{4} \sim 10^{8}$ 的增强 效应 ${ }^{[6]}$. 实际上, 表面增强效应除 EM增强外, 化学增强 机理(chemical enhancement, CE)亦发挥了重要作用, 其 源于金属和吸附分子波函数的重叠, 主要分为三 类 ${ }^{[6 \sim 10]}$ : (1)共振拉曼机理. 当入射光的能量与分子内部 所需的跃迁能相匹配时, 分子就会发生共振拉曼散射, 其拉曼强度与跃迁偶极矩、激发态弛豫时间有关. 而跃 迁偶极矩、激发态弛豫时间会受到金属基底的影响，因 此也将这种共振拉曼效应归为化学增强机理, 一般该种 增强机理对 SERS 增强效应的贡献为 $10^{3} \sim 10^{6}$. (2) 电荷 转移机理. 当入射光的能量与体系内部电荷转移所需能 量相匹配时, 金属基底和其表面化学吸附的探针分子之 间可以发生一种类共振的电荷转移过程, 该过程可以增 大吸附分子的极化率, 从而增强分子的拉曼信号 ${ }^{[7]}$. 一 般该增强机理对 SERS 增强效应的贡献约为 $10^{3}[8]$. (3) 非共振化学机理. 当分子靠近基底表面, 分子和基底之 间的成键与化学吸附作用会改变分子的电子结构和极 化率，从而产生增强效应 ${ }^{[9,10]}$.一般该种增强机理对 SERS 增强效应的贡献约为 $10^{[8]}$.

相对于 EM 机理, 人们对于 CE 增强机理的本质还 不是十分清晰. 对于增强机理的研究依赖于使用的 SERS 基底, 目前常用的 SERS 基底主要有少数纳米级 的贵金属型基底、半导体型基底和金属-半导体异质结 型基底. 贵金属型、金属-半导体异质结型 SERS 基底中 $\mathrm{EM}$ 和 CE 并存, 通常难以区分它们各自的贡献, 而对于 宽禁带半导体而言, 其表面等离子体的共振频率分布在 红外光 ${ }^{[11]}$, 因此可以通过调节激发波长, 使其只产生 $\mathrm{CE}$, 而不涉及 $\mathrm{EM}$, 为单独研究 $\mathrm{CE}$ 提供了一个很好的 平台.

作为一种宽禁带半导体纳米材料, $\mathrm{ZnO}$ 具有许多优 良的性质如高效率的光催化性能及热电、压电效应 ${ }^{[12,13]}$. 此外, 六棱柱型纳米 $\mathrm{ZnO}$ 因其材料自身及形貌特性对 光具有增益效应, 在光照射下, 通过谐振腔模式可发射 激光, 产生光波导效应 ${ }^{[14,15]}$. 因此, 纳米 $\mathrm{ZnO}$ 材料在苂 光光谱及拉曼光谱中应用非常广泛.

虽然因 $\mathrm{ZnO}$ 优越的光学性能而在荧光分析中实现 了 DNA 和蛋白质等物质的高灵敏度检测 ${ }^{[16]}$; 而对于 SERS 而言, 早期的研究报道表明 $\mathrm{ZnO}$ 胶体的 SERS 效
应非常微弱, 其以染料 D266 为探针在 $\mathrm{ZnO}$ 胶体上进行 拉曼检测, 发现增强因子只约为 $50^{[17]}$; 随后对于纳米 $\mathrm{ZnO}$ 材料在 SERS 中的应用展开了多方面的研究, 如 Sun 等 ${ }^{[18]}$ 以对颈基苯甲酸 (para-mercaptobenzoic acid, MBA)和 4-颈基吡啶(4-mercaptopyridine, 4-MPY)两种分 子为探针, 通过研究其在不同粒径纳米 $\mathrm{ZnO}$ 颗粒上拉 曼信号的强度与 $\mathrm{ZnO}$ 基底尺寸的关系, 得出了纳米 $\mathrm{ZnO}$ 的 SERS 效应具有尺寸依赖性, 但却忽略了表面增强因 子(enhancement factor, $E F$ ) 的计算; Wang 等 ${ }^{[11]}$ 通过检测 基底上 4-MPY 的 SERS 强度，计算得到 $20 \mathrm{~nm}$ 球形 $\mathrm{ZnO}$ 的 $E F$ 约为 $10^{3}$, 并将这种增强效应归因于半导体和吸附 分子之间的电子转移; Wang 等 ${ }^{[19]}$ 在非晶态 $\mathrm{ZnO}$ 纳米笼 上检测到探针分子的特征 SERS 谱峰, 并计算发现其 $E F$ 可达 $10^{5}$, 实际上却忽略了以 $\mathrm{Cu}_{2} \mathrm{O}$ 纳米立方体为模板制 备的非晶态 $\mathrm{ZnO}$ 纳米笼中残留少量的具有较强 SERS 增强效应的 $\mathrm{Cu}_{2} \mathrm{O}$ 纳米颗粒的贡献 ${ }^{[20]}$, 致使其计算的 $E F$ 偏高. 迄今为止, 仍然缺乏对于 $\mathrm{ZnO}$ 表面 SERS 效应的 系统研究, 因此亟待深入挖掘半导体 $\mathrm{ZnO}$ 的 SERS 增强 机理, 有利于拓展 SERS 研究至 $\mathrm{ZnO}$ 表面.

本文利用高温分解法制备了具有高比表面的扫帚 状 $\mathrm{ZnO}$ 纳米棒集合体, 对其进行系统表征, 以取代基性 质不同的对硝基苯硫酚 (para-nitrophenylthiophenol, PNTP)、苯硫酚 (thiophenol, TP)、对氨基苯硫酚 (para-aminothiophenol, PATP) 为探针分子, 系统研究了 $\mathrm{ZnO}$ 纳米棒集合体表面的 SERS 效应，估算了该 $\mathrm{ZnO}$ 纳 米棒集合体的 SERS 增强因子, 并提出了可能的增强机 理.

\section{2 结果与讨论}

\section{1 扫帚状纳米 $\mathrm{ZnO}$ 的制备与表征}

通过 $\left(\mathrm{CH}_{3} \mathrm{COO}\right)_{2} \mathrm{Zn}$ 高温分解, 成功地制备了纳米 $\mathrm{ZnO}$, 其形貌如图 $1 \mathrm{a}$ 和 $1 \mathrm{~b}$ 所示. 由图可知, 纳米 $\mathrm{ZnO}$ 呈扫帚状, 结构与形貌均一, 是多个 $\mathrm{ZnO}$ 纳米棒的集合 体. 该纳米结构的总高度约 $740 \mathrm{~nm}$, 上部高度约 570 $\mathrm{nm}$, 最大宽度约 $860 \mathrm{~nm}$, 下部高度约 $170 \mathrm{~nm}$, 宽度约 $170 \mathrm{~nm}$. 单根纳米 $\mathrm{ZnO}$ 棒的直径约 $16.6 \mathrm{~nm}$, 长度约 570 $\mathrm{nm}$. 由图 1c 可知, $365 \mathrm{~nm}$ 处观察到明显的紫外吸收峰, 因 $\mathrm{ZnO}$ 的禁带宽度约为 $3.37 \mathrm{eV}$, 所以该吸收峰是由纳 米 $\mathrm{ZnO}$ 的带-带跃迁引起的. 由图 $1 \mathrm{~d}$ 的 XRD 可知, 衍 射峰的位置与 JCPDS: 36-1451 标准峰相同, 表明 ZnO 为纤锌矿六方相结构; 衍射峰的峰型较尖锐, 表明 $\mathrm{ZnO}$ 具有较高的结晶度; 未检测到其他衍射峰, 表明合成的 纳米 $\mathrm{ZnO}$ 纯净, 无杂质; 且该 $\mathrm{ZnO}$ (002) 晶面衍射峰的 相对高度与标准 JCPDS 卡数据相比较高, 表明纳米 $\mathrm{ZnO}$ 棒优先沿着 $<001>$ 方向生长. 其具体过程如下: 反应开始时, 溶液中覆盖剂油胺的浓度相对较高, 其长 烷基链可以在较大程度上络合 $\mathrm{ZnO}$ 的各个晶面，因此 首先生长成立方柱状体; 随着反应的进行，已生成的立 

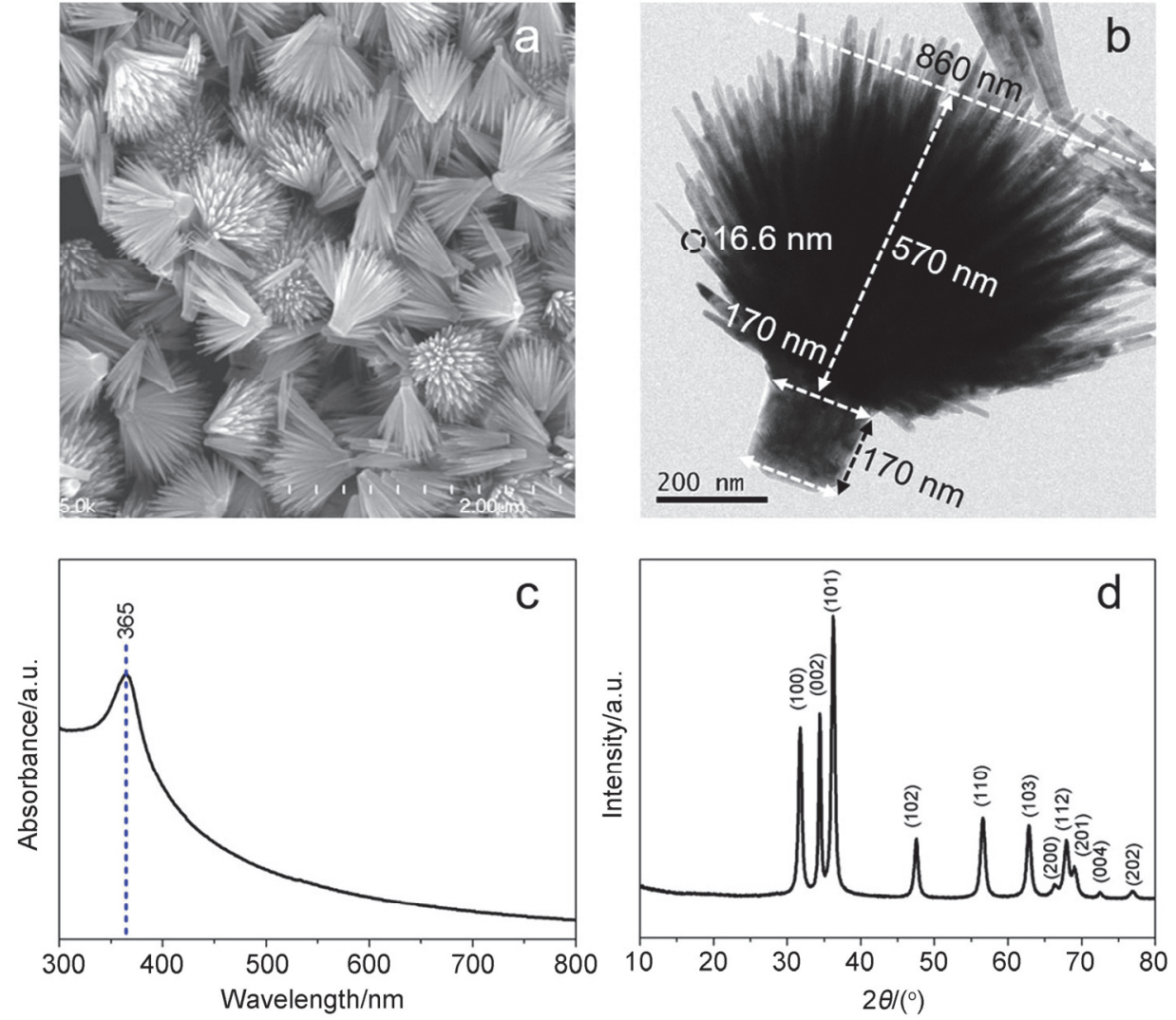

图 $1 \mathrm{ZnO}$ 的扫描电镜图(a), 透射电镜图(b), 紫外可见吸收光谱图(c)和 X 射线粉末衍射谱图(d)

Figure 1 SEM (a), TEM (b) images, UV-Vis absorption spectrum (c) and XRD pattern (d) of as-prepared ZnO nanoparticles

方柱状 $\mathrm{ZnO}$ 可作为后面纳米结构生长的种子层, 溶液 中生成的随机取向的 $\mathrm{ZnO}$ 晶核下落到种子层表面并缓 慢生长, 但由于此时溶液中油胺的浓度较低, 其长烷基 链不足以络合 $\mathrm{ZnO}$ 的各个晶面, 而 $\mathrm{ZnO}$ 的(001)面是一 个非常不稳定的极性面, 其较高的表面能使得 $\mathrm{ZnO}$ 在 $<001>$ 方向的生长速率大大增加, 最终形成一维棒状 结构; 且由于 $\mathrm{ZnO}$ 晶核的随机取向, 致使 $\mathrm{ZnO}$ 纳米棒阵 列在立方柱状 $\mathrm{ZnO}$ 种子层表面参差不齐呈无序状排列.

由前面的 SEM、TEM 图可见, $\mathrm{ZnO}$ 呈棒状延伸生长, 因此其裸露的表面较大, 为分子在表面的吸附或反应提 供了大量的位置. 纳米材料的比表面积影响分子最终的 吸附量, 因此, 对扫帚状纳米 $\mathrm{ZnO}$ 进行比表面积测试, 其 $\mathrm{N}_{2}$ 吸附-脱附等温曲线如图 2 所示. 通过 BET 模型计 算可知, 扫帚状纳米 $\mathrm{ZnO}$ 的比表面积为 $24.31 \mathrm{~m}^{2} / \mathrm{g}$.

\section{2 扫帚状纳米 ZnO 的 SERS 性能研究}

众所周知，巨大的 SERS 增强效应通常来源于贵金 属纳米级的粗粘表面. 目前, SERS 基底材料已由传统贵 金属、过渡金属等扩展到纳米半导体材料. 如 Yang 等 ${ }^{[21]}$ 在尺寸为 $10 \mathrm{~nm}$ 左右的半导体 $\mathrm{TiO}_{2}$ 上检测到了探针分 子 MBA、4-MPY 和 PATP 的拉曼信号, 认为这是 $\mathrm{TiO}_{2}$ 价带上的电子在激光激发下转移至其自身的表面态能 级, 进一步转移至探针分子所致, 且探针分子颈基对位
基团的吸电子能力越强 $\left(\mathrm{C}-\mathrm{COOH}>\mathrm{N}>\mathrm{C}-\mathrm{NH}_{2}\right)$, 其拉曼 信号增强越强.

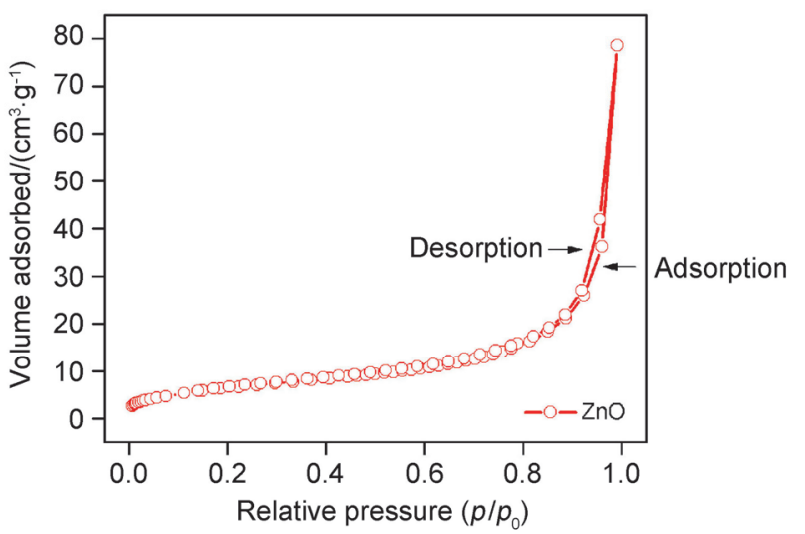

图 2 扫帚状纳米 $\mathrm{ZnO}$ 的 $\mathrm{N}_{2}$ 吸附-脱附曲线

Figure 2 The $\mathrm{N}_{2}$ adsorption-desorption curve of the broom-like $\mathrm{ZnO}$ nanoparticles

为深入研究半导体氧化物 SERS 效应增强机理的来 源, 以颈基对位取代基吸电子能力不同的 PNTP、TP 和 PATP 为探针分子, 研究了均匀性良好的 $\mathrm{ZnO}$ 基底(详见 支持信息)表面随激发波长变化的 SERS 光谱(图 3), 并 研究了其增强机理. 由图 3a 可知, $532 \mathrm{~nm}$ 激光下, 在空 白的 $\mathrm{ZnO}$ 表面仅检测到位于 $1100 \mathrm{~cm}^{-1}$ 左右较宽的谱峰, 
其主要来自于 $\mathrm{ZnO}$ 本征振动峰. 当分别吸附 PNTP、TP 及 PATP 之后, 分别观察到了这三种分子的特征振动峰, 如观察到位于 1085、1342 和 $1576 \mathrm{~cm}^{-1}$ 处归属于 PNTP 的 $v_{\mathrm{CS}} 、 v_{\mathrm{S}}$ (NO2) 和苯环上 $v_{\mathrm{CC}}$ 的特征谱峰 ${ }^{[22]}$, 位于 998 、 1035 和 $1580 \mathrm{~cm}^{-1}$ 处归属于 TP 苯环 $\delta_{\mathrm{r}-\mathrm{i}-\mathrm{d}}+v_{\mathrm{CC}} 、 v_{\mathrm{CC}}+\delta_{\mathrm{CH}}$ 和 $v_{\mathrm{CC}}$ 的特征谱峰 ${ }^{[23]}$, 或位于 $1592 \mathrm{~cm}^{-1}$ 处归属于 PATP 苯环上 $v_{\mathrm{CC}}$ 的特征谱峰 ${ }^{[24]}$. 对比三种分子分别归属于 $v_{\mathrm{CC}}$ 处谱峰的积分强度发现, 三种分子 SERS 信号绝对强度 的顺序为 PNTP $>$ PATP $>$ TP.

当采用 $638 \mathrm{~nm}$ 激光激发时, 同样观察到了 PNTP、 TP 及 PATP 的特征谱峰. 对比三种分子分别归属于 $v_{\mathrm{CC}}$ 处谱峰的积分强度发现, 三种分子 SERS 信号绝对强度 的顺序为 PNTP $>$ PATP $>$ TP. 虽然三种分子的绝对强度 不同, 但由于不同分子本身的散射截面不同, 半导体 $\mathrm{ZnO}$ 型 SERS 基底表面这三种分子的 SERS 信号绝对强 度也会有所不同，因此并不能直接推测在相同激发线下 这三种分子增强效应的强弱, 尚需通过增强因子的计算 方能判断增强效应的差别.
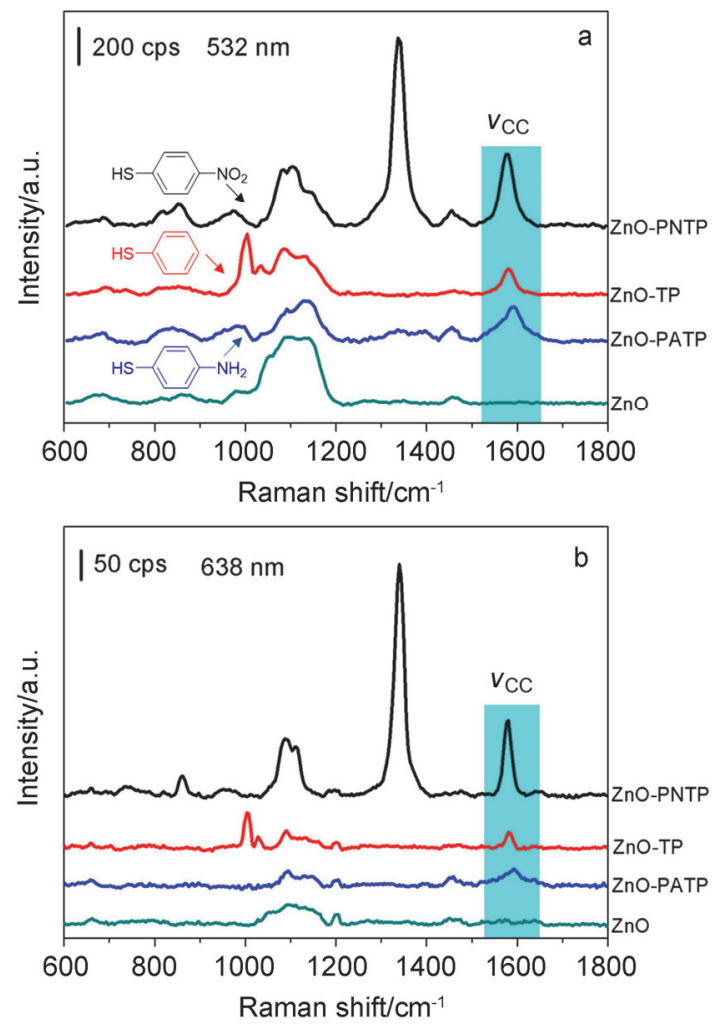

图 $3532 \mathrm{~nm}$ (a)和 $638 \mathrm{~nm}$ (b)激光下, PNTP, TP, PATP 吸附在扫帚状 纳米 $\mathrm{ZnO}$ 上的 SERS 谱图

Figure 3 SERS spectra of PNTP, TP and PATP adsorbed on the processed $\mathrm{ZnO}$ nanoparticles with $532 \mathrm{~nm}$ (a) and $638 \mathrm{~nm}$ (b) laser excitation

\section{3 扫帚状纳米 $\mathrm{ZnO}$ 上的表面增强因子}

以上实验结果中并未发现 SERS 强度与探针分子颈 基对位基团吸电子能力强弱之间的关系. 表面增强因子 $(E F)$ 是衡量 SERS 基底活性的标准 ${ }^{[25]}$, 因此有必要计算
这三种探针分子在纳米 $\mathrm{ZnO}$ 上的 $E F . E F$ 指的是分子数 相同时, 目标分子在基底上的 SERS 信号与这些分子常 规拉曼信号之间的比值，可表示为:

$$
E F=\frac{I_{\text {surf }}}{I_{\text {bulk }}} \times \frac{N_{\text {bulk }}}{N_{\text {surf }}}
$$

$I_{\text {surf }}$ 和 $I_{\mathrm{bulk}}$ 分别是指目标分子同一振动模式在 SERS 基底 与溶液中拉曼信号的积分强度, 其值可直接从相应拉曼 谱峰的强度积分得到. $N_{\text {surf }}$ 和 $N_{\text {bulk }}$ 分别是指同一激发光 下 SERS 基底表面吸附的分子数与溶液中的分子数.

本文分别选择归属于三种探针分子苯环上 $v_{\mathrm{CC}}$ 特征 谱峰的强度积分得到相应的 $I_{\text {surf }}$ (图 3) 和 $I_{\text {bulk. }}$. 图 4 是以 二氯甲烷为溶剂的不同浓度探针分子的本征拉曼谱图. 由于这些分子的水溶性较差, 采用二氯甲烷作为溶剂主 要是考虑到其在 $1500 \sim 1800 \mathrm{~cm}^{-1}$ 波数范围内并无拉曼 特征峰，因此对于探针分子苯环上 $v_{\mathrm{CC}}$ 特征谱峰的检测 并无干扰，通过计算溶液中探针分子 $v_{\mathrm{CC}}$ 特征谱峰的积 分强度可获得相应的 $I_{\text {bulk. }}$.
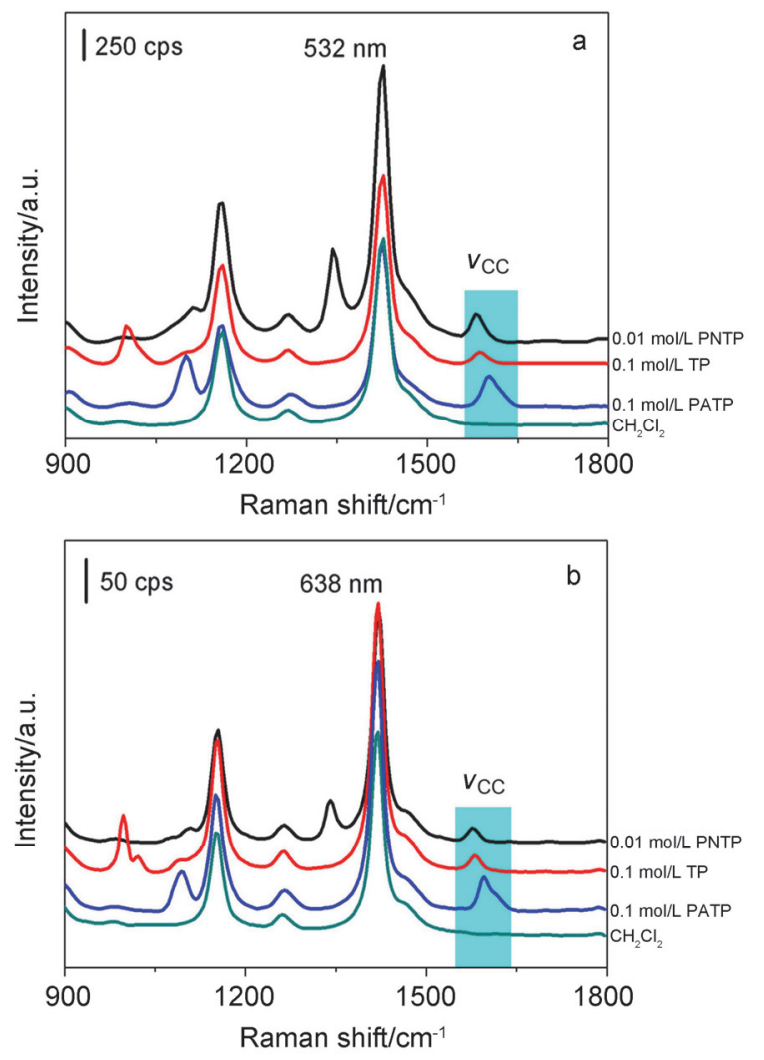

图 $4532 \mathrm{~nm}$ (a)和 $638 \mathrm{~nm}$ (b)激光下, $0.01 \mathrm{~mol} / \mathrm{L}$ PNTP 二氯甲烷溶液, $0.1 \mathrm{~mol} / \mathrm{L} \mathrm{TP}$ 二氯甲烷溶液和 $0.1 \mathrm{~mol} / \mathrm{L}$ PATP 二氯甲烷溶液的拉曼谱 图

Figure 4 Raman spectra of $0.01 \mathrm{~mol} / \mathrm{L}$ PNTP dichloromethane solution, $0.1 \mathrm{~mol} / \mathrm{L} \mathrm{TP}$ dichloromethane solution and $0.1 \mathrm{~mol} / \mathrm{L}$ PATP dichloromethane solution under $532 \mathrm{~nm}$ (a) and $638 \mathrm{~nm}$ (b) laser irradiation

对于 $N_{\text {surf }}$ 的计算(详见支持信息), 通过将单个扫帚 状纳米 $\mathrm{ZnO}$ 简化为相应的数学模型, 计算得到激光光 斑下纳米 $\mathrm{ZnO}$ 的表面积. 这三种分子在 $\mathrm{ZnO}$ 表面的吸 
附均是垂直构型, 其垂直表面积直接影响 $E F$ 的计算, 但并没有相关的文献数据, 虽然这三种分子的表面积存 在一定差距, 但考虑到 PNTP、TP、PATP 与模型分子吡 啶均为六元环状结构, 因此可用单个吡啶分子的表面积 $0.25 \mathrm{~nm}^{2}[26]$ 代替这三种分子进行计算.

对于 $N_{\text {bulk }}$ 的计算(详见支持信息), 必须考虑共聚焦 拉曼光谱仪器的光学特点, 计算得到不同激光下溶液中 的分子数.

表 1 列出了通过以上方法计算得到的纳米 $\mathrm{ZnO}$ 表 面三种探针分子在不同激光下的 $E F$ 数值. 从表中可知, $\mathrm{ZnO}$ 上三种探针分子的 $E F$ 总体相差较小, 其数值均在 40 以内. 与 PNTP 相比, TP、PATP 的 $E F$ 数值较大, 且 更为接近.

表 1 不同激光下纳米 $\mathrm{ZnO}$ 上三种探针分子的表面增强因子

Table 1 Surface enhancement factor of three probe molecules on $\mathrm{ZnO}$ nanoparticles under different lasers

\begin{tabular}{cccc}
\hline \multirow{2}{*}{ Laser/nm } & \multicolumn{3}{c}{$E F$} \\
\cline { 2 - 4 } & PNTP & TP & PATP \\
\hline 532 & 9.36 & 35.42 & 27.33 \\
638 & 14.01 & 25.23 & 22.36 \\
\hline
\end{tabular}

\section{4 纳米 $\mathrm{ZnO}$ 的 SERS 增强机理}

目前普遍接受的 SERS 增强机理主要有两种: 源于 表面等离子体共振的 $\mathrm{EM}$ 机理与源于金属和吸附分子波 函数重叠的 $\mathrm{CE}$ 机理. 对于宽禁带半导体纳米 $\mathrm{ZnO}$ 而言, 其表面等离子体的共振频率位于红外区 ${ }^{[11]}$, 而本实验中 所用的激发线是 $532 \mathrm{~nm}$ 和 $638 \mathrm{~nm}$, 远离红外区, 因此 正如文献报道的, SERS 效应主要的贡献来自 CE 机理.

通常 CE 机理主要来源于共振、电荷转移以及非共 振三种增强效应. 纳米 $\mathrm{ZnO}$ 是一种典型的 $\mathrm{n}$ 型半导体, 其表面具有丰富的表面缺陷，如表面氧空位等，这些表 面缺陷可以束缚电子形成表面态能级 $\left(E_{\mathrm{SS}}\right)^{[21]}$. 实验中 三种探针分子通过 $\mathrm{Zn}-\mathrm{S}$ 键化学吸附于纳米 $\mathrm{ZnO}$ 表 面 ${ }^{[19]}$. 激光光子作用下, $\mathrm{ZnO}$-吸附分子体系可能发生的 电子跃迁过程有三种：(1)吸附分子最高已占轨道 (HOMO)上的电子被激发跃迁至其自身的最低未占轨道 (LUMO), 发生共振拉曼散射, 产生共振增强效应; (2)半 导体 $\mathrm{ZnO}$ 价带( $\mathrm{VB}$ )上的电子被激发, 转移至其自身的 $E_{\mathrm{SS}}$, 进而转移至吸附分子, 发生光子驱动下的类共振 拉曼过程, 产生 SERS 增强效应, 如 Zhao 等 ${ }^{[21]}$ 报道的 4-MPY 吸附在 $\mathrm{TiO}_{2}$ 表面的 SERS 增强来源于此电荷转 移效应; (3)吸附分子 $\mathrm{HOMO}$ 上的电子被激发转移至半 导体的 $E_{\mathrm{SS}}$ 或导带 $(\mathrm{CB})$, 进而产生 SERS 增强效应.

本文通过密度泛函理论, 计算得到三种探针分子的 前线轨道 (详见支持信息), 结果发现三种分子的 HOMO-LUMO 带隙均大于 $4 \mathrm{eV}$, 而实验中所用激光的 能量最高为 $2.26 \mathrm{eV}$, 因此无法发生(1)过程; $\mathrm{ZnO}$ 的 $\mathrm{VB}$ 和 $\mathrm{CB}$ 分别为 $-7.7 \mathrm{eV}$ 和 $-4.3 \mathrm{eV}$ (相对于真空能级, 下 同 $)^{[11]}$. 激光照射下, $\mathrm{ZnO}$ 的 $\mathrm{VB}$ 中的电子最高可被激发
至 $-5.44 \mathrm{eV}$ 的能级, 其与吸附分子 LUMO 轨道的能级 依然相差较大，因此无法发生(2)过程; 当吸附分子为 TP 或 PATP 时, 其 HOMO 轨道中的电子最低可被激发 转移至 $-4.06 \mathrm{eV}$ 的能级, 其与 $\mathrm{ZnO}$ 的 $\mathrm{CB}(-4.3 \mathrm{eV})$ 较 为接近, 电子进而可转移至 $\mathrm{ZnO}$ 的 $\mathrm{CB}$ 产生光子驱动的 电荷转移 SERS 增强效应, (3)过程可以发生. 激光光子 能量越高, TP 和 PATP 的 SERS 增强效应越强, 这与我们 计算得到的 TP、PATP 的 EF 结果相吻合. 与 PNTP 相 比，探针分子 TP 和 PATP 具有更为相近的电子能级，其 $E F$ 数值应更为接近, 这也与我们计算得到的 TP、PATP 的 $E F$ 结果相吻合; 而当吸附分子为 PNTP 时, 其 $\mathrm{HOMO}$ 轨道中的电子最高可被激发至 $-4.48 \mathrm{eV}$, 其与 $\mathrm{ZnO}$ 的 $\mathrm{CB}(-4.3 \mathrm{eV})$ 仍有一定差距, 激发后电子所处能 级与 $\mathrm{ZnO}$ 能级的匹配性较差而使(3)过程的发生机率较 低, 因此半导体 ZnO 对 PNTP 的 SERS 增强效应主要来 源于非共振化学增强机理. 探针分子 PNTP 通过 $\mathrm{Zn}-\mathrm{S}$ 键化学吸附于 $\mathrm{ZnO}$ 的表面, $\mathrm{ZnO}$ 的存在改变了表面吸附 分子 PNTP 的电子结构与极化率，从而产生了 SERS 增 强效应，根据文献报道该种机理产生的增强效应通常约

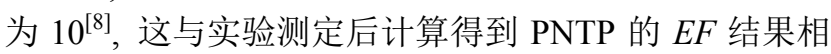
吻合.

综合以上分析, 扫帚状纳米 $\mathrm{ZnO}$ 上不同吸附分子 的 SERS 增强效应可能来源于不同的增强机理：对于 TP 与 PATP 分子来说, $\mathrm{ZnO}$ 的 SERS 增强效应可能主要来源 于因发生吸附分子到半导体的电子跃迁过程而产生的 电荷转移机理; 对于 PNTP 分子来说, 由于硝基显著的 吸电子效应，导致其 HOMO、LUMO 能级变化较大，光 子驱动的电荷转移几乎不可能发生, $\mathrm{ZnO}$ 对其的 SERS 增强效应可能主要来源于因分子与基底间的相互作用 引起 PNTP 分子电子结构与极化率改变而产生的非共振 化学机理. 且在 $532 \mathrm{~nm}$ 激发光作用下, 光子驱动的电荷 转移增强效应较 $638 \mathrm{~nm}$ 激发光更强, 这主要是前者更 高的光子能量导致发生电荷转移的可能性提高所致. 此 外, 在半导体表面光子驱动的电荷转移增强效应较贵金 属表面的低, 这与吸附分子和纳米 $\mathrm{ZnO}$ 间的电荷转移 速率较慢有关, 文献报道分子与 $\mathrm{TiO}_{2}$ 间的电荷转移速 率较纳米 $\mathrm{ZnO}$ 的快 1 个数量级以上 ${ }^{[27]}$, 因此 $\mathrm{TiO}_{2}$ 表面 因光子驱动电荷转移所致的增强效应也明显高于 $\mathrm{ZnO}$ 表面，而分子与金属间光子驱动电荷转移的速率更快, 因此其 SERS 增强因子通常可以达到三个数量级 ${ }^{[8]}$, 明 显大于纳米 $\mathrm{TiO}_{2}$ 和 $\mathrm{ZnO}$.

\section{3 结论}

通过 $\left(\mathrm{CH}_{3} \mathrm{COO}\right)_{2} \mathrm{Zn}$ 高温分解法获得了形貌新颖、尺 寸均一的扫帚状纳米 $\mathrm{ZnO}$, 对其进行 SEM、TEM、 UV-Vis、XRD、BET 表征, 通过在扫帚状纳米 $\mathrm{ZnO}$ 上 修饰 PNTP、TP、PATP 分子, 系统地研究了该 $\mathrm{ZnO}$ 在 $532 \mathrm{~nm}$ 和 $638 \mathrm{~nm}$ 激光下的 SERS 行为, 估算了各自的 
$E F$. 对于因强吸电子基团取代的 PNTP 来说, 其能级与 $\mathrm{ZnO}$ 的匹配性较差, 约 10 倍的增强效应主要来自于因 吸附而导致电子结构变化后分子极化率的改变所产生 的非共振增强机理. 而对于 TP 和 PATP 来说, 其 $20 \sim 35$ 倍的 SERS 增强则是由非共振增强效应和光子驱动的电 荷转移效应共同贡献所致, 其中光子能量越高, SERS 增 强效应越强. 且因分子与 $\mathrm{ZnO}$ 间电荷转移速率较慢导 致 $\mathrm{ZnO}$ 上光子驱动的电荷转移增强效应较 $\mathrm{TiO}_{2}$ 和金属 弱 $1 \sim 2$ 个数量级. 本文的研究结果为深入探讨半导体 SERS 增强机理以及分离化学增强贡献提供了新途径.

\section{4 实验部分}

\section{1 扫帚状纳米 $\mathrm{ZnO}$ 的制备}

在 Tahir 等 ${ }^{[28]}$ 报道的制备纳米 $\mathrm{ZnO}$ 的方法上稍作修 改: 将 $0.0947 \mathrm{~g}\left(\mathrm{CH}_{3} \mathrm{COO}\right)_{2} \mathrm{Zn}$ 加入到 $6 \mathrm{~mL}$ 苯甲醇与 3 $\mathrm{mL}$ 油胺的混合溶液中, 超声 $10 \mathrm{~min}$, 使 $\left(\mathrm{CH}_{3} \mathrm{COO}\right)_{2} \mathrm{Zn}$ 完全溶解. 然后, 缓慢升温至 $180{ }^{\circ} \mathrm{C}$, 在此温度下反应 $20 \mathrm{~min}$. 反应完全后, 将混合液移出并自然冷却到室温, 加入适量无水乙醇, 多次离心清洗, 并将所得的白色沉 淀重新分散在 $5 \mathrm{~mL}$ 无水乙醇中, 待用.

\subsection{SERS 基底的制备}

取 $20 \mu \mathrm{L}$ 新制得的扫帚状纳米 $\mathrm{ZnO}$ 乙醇溶液滴加在 $4 \mathrm{~mm} \times 4 \mathrm{~mm}$ 的硅片上, 真空干燥后将其分别浸泡在浓 度为 $1 \mathrm{mmol} / \mathrm{L}$ 的 PNTP、TP、PATP 的乙醇溶液中 $30 \mathrm{~min}$, 使纳米 $\mathrm{ZnO}$ 表面修饰上探针分子, 大量无水乙醇清洗, 去除物理吸附的探针分子, 待用.

\section{References}

[1] Halas, N. J.; Moskovits, M. MRS Bull. 2013, 38, 607.

[2] Wang, M.; Yan, X.; Wei, D.; Liang, L.; Wang, Y. Acta Chim. Sinica 2019, 77, 184. (王猛, 间昕, 韦德泉, 梁兰菊, 王岳平, 化学学报, 2019, 77, 184.)

[3] Kelly, K. L.; Coronado, E.; Zhao, L. L.; Schatz, G. C. J. Phys. Chem. $B$ 2003, 107, 668 .
[4] Stiles, P. L.; Dieringer, J. A.; Shah, N. C.; van Duyne, R. R. Annu. Rev. Anal. Chem. 2008, 1, 601.

[5] Halas, N. J.; Lal, S.; Chang, W. S.; Link, S.; Nordlander, P. Chem. Rev. 2011, 111, 3913

[6] Wu, D. Y.; Liu, X. M.; Duan, S.; Xu, X.; Ren, B.; Lin, S. H.; Tian, Z Q. J. Phys. Chem. C 2008, 112, 4195.

[7] Tian, Z. Q.; Ren, B.; Wu, D. Y. J. Phys. Chem. B 2002, 106, 9463.

[8] Zhao, L. L.; Jensen, L.; Schatz, G. C. J. Am. Chem. Soc. 2006, 128, 2911.

[9] Tian, Z. Q.; Ren, B. Annu. Rev. Phys. Chem. 2004, 55, 197.

[10] Wu, D. Y.; Li, J. F.; Ren, B.; Tian, Z. Q. Chem. Soc. Rev. 2008, 37, 1025.

[11] Wang, Y. F.; Ruan, W. D.; Zhang, J. H.; Yang, B.; Xu, W. Q.; Zhao, B.; Lombardi, J. R. J. Raman Spectrosc. 2009, 40, 1072.

[12] Klingshirn, C.; Fallert, J.; Zhou, H.; Sartor, J.; Thiele, C.; Maier Flaig, F.; Schneider, D.; Kalt, H. Phys. Status Solidi B 2010, 247, 1424.

[13] Yang, P. D.; Yan, R. X.; Fardy, M. Nano Lett. 2010, 10, 1529.

[14] Chu, S.; Wang, G. P.; Zhou, W. H.; Lin, Y. Q.; Chernyak, L.; Zhao, J. Z.; Kong, J. Y.; Li, L.; Ren, J. J.; Liu, J. L. Nat. Nanotechnol. 2011, 6,506 .

[15] Zhao, D.; Zhang, X. X.; Dong, H. B.; Yang, L. J.; Zeng, Q. S.; Li, J. Z.; Cai, L.; Zhang, X.; Luan, P. S.; Zhang, Q.; Tu, M.; Wang, S.; Zhou, W. Y.; Xie, S. S. Nanoscale 2013, 5, 4443.

[16] Dorfman, A.; Kumar, N.; Hahm, J. Langmuir 2006, 22, 4890.

[17] Wen, H.; He, T. J.; Xu, C. Y.; Zuo, J.; Liu, F. C. Mol. Phys. 1996, 88, 281.

[18] Sun, Z. H.; Zhao, B.; Lombardi, J. R. Appl. Phys. Lett. 2007, 91, 221106.

[19] Wang, X. T.; Shi, W. X.; Jin, Z.; Huang, W. F.; Lin, J.; Ma, G. S.; Li, S. Z.; Guo, L. Angew. Chem., Int. Ed. 2017, 56, 9851.

[20] Lin, J.; Shang, Y.; Li, X. X.; Yu, J.; Wang, X. T.; Guo, L. Adv. Mater 2016, 29, 1.

[21] Yang, L. B.; Jiang, X.; Ruan, W. D.; Zhao, B.; Xu, W. Q.; Lombardi, J. R. J. Phys. Chem. C 2008, 112, 20095.

[22] Dong, B.; Fang, Y. R.; Xia, L. X.; Xu, H. X.; Sun, M. T. J. Raman Spectrosc. 2011, 42, 1205.

[23] Blum, C.; Opilik, L.; Atkin, J. M.; Braun, K.; Käemmer, S. B.; Kravtsov, V.; Kumar, N.; Lemeshko, S.; Li, J. F.; Luszcz, K.; Maleki, T.; Meixner, A. J.; Minne, S.; Raschke, M. B.; Ren, B.; Rogalski, J.; Roy, D.; Stephanidis, B.; Wang, X.; Zhang, D.; Zhong, J. H.; Zenobi, R. J. Raman Spectrosc. 2014, 45, 22.

[24] Fang, Y. R.; Li, Y. Z.; Xu, H. X.; Sun, M. T. Langmuir 2010, 26, 7737.

[25] Guo, Q. H.; Xu, M. M.; Yuan, Y. X.; Gu, R. A.; Yao, J. L. Langmuir 2016, 32, 4530 .

[26] Conway, E. B.; Mathieson, J.; Dhar, P. H. J. Phys. Chem. 1974, 78, 1226.

[27] Wei, H.; Luo, J. W.; Li, S. S.; Wang, L. W. J. Am. Chem. Soc. 2016, 138,8165 .

[28] Tahir, M. N.; Natalio, F.; Cambaz, M. A.; Panthöfer, M.; Branscheid, R.; Kolb, U.; Tremel, W. Nanoscale 2013, 5, 9944. 